\title{
Establishment of the Steady State in Glucose-limited Chemostat Cultures of Klebsiella pneumoniae
}

\author{
By M. RUTGERS, ${ }^{1,2 *}$ M. J. TEIXEIRA DE MATTOS, ${ }^{3}$ P. W. POSTMA ${ }^{1}$ \\ AND K. VAN DAM ${ }^{1}$ \\ ${ }^{* 1}$ Laboratory of Biochemistry, University of Amsterdam, P O Box 20151, 1000 HD Amsterdam, \\ The Netherlands \\ ${ }^{2}$ Biotechnology Centre, University of Amsterdam, The Netherlands \\ ${ }^{3}$ Laboratory for Microbiology, University of Amsterdam, Nieuwe Achtergracht 127, \\ 1018 WS Amsterdam, The Netherlands
}

(Received 10 July 1986; revised 22 October 1986)

To investigate the relationship between growth rate and concentration of the nutrient that limits growth, 'Klebsiella aerogenes' NCTC 418 ( $K$. pneumoniae) was grown in a glucose-limited chemostat. The actual time required to establish a steady-state glucose concentration exceeded that expected theoretically. Apparently, there is a long-term adaptation of the cells to nutrient limitation. As yet, it is not clear whether this has a phenotypic or genetic origin. In the final steady state, the dependence of the growth rate on glucose concentration could be mathematically described equally well by a hyperbolic and by a logarithmic function.

\section{INTRODUCTION}

In order to come to a better understanding of the behaviour of bacterial cultures, various descriptions of bacterial growth and product formation have been developed (for a review see van Dam et al., 1987).

In the classical approach, initiated mainly by the work of Monod (1950), bacterial cells are considered as catalytic units, the metabolic activities of which can be described by simple Michaelis-Menten kinetics. Thus, under nutrient-limited growth conditions - as is the case in chemostat cultures - a hyperbolic relationship between the growth rate and the steady-state concentration of the limiting substrate is expected. This relationship is solely dependent on the kinetic parameters $K_{\mathrm{s}}$ and $\mu_{\max }$, both properties of the organism:

$$
\mu=\mu_{\max } \frac{\bar{s}}{K_{\mathrm{s}}+\bar{s}}
$$

(where $\mu$ is the specific growth rate, $\mu_{\max }$ the maximal growth rate, $\bar{s}$ the concentration of the limiting substrate in the culture and $K_{\mathrm{s}}$ the overall affinity constant for the substrate that limits the growth rate). This kinetic approach implies that the relationship between $\mu$ and $\bar{s}$ is unique at a defined set of environmental conditions.

Bacterial growth has also been described on the basis of concepts that originate from nonequilibrium thermodynamics (Westerhoff et al., 1982). In this description it is supposed that the rates of metabolism (i.e. specific respiration or carbon substrate uptake rates) are governed by the differences in free energy between substrates and products $(\Delta G)$. The following relation should apply:

$$
J_{\mathrm{a}}=L_{\mathrm{aa}} \cdot \Delta G_{\mathrm{a}}+L_{\mathrm{ac}} \cdot \Delta G_{\mathrm{c}}
$$

Abbreviations: $\mu$, specific growth rate $; \mu_{\max }$, maximal growth rate; $D$, dilution rate $; J_{\mathrm{a}}$, rate of anabolism $; J_{\mathrm{c}}$, rate of catabolism; $K_{\mathrm{s}}$, overall affinity constant for substrate limiting growth rate; $L$, proportionality constant; $\bar{s}$, concentration of limiting substrate; $V_{\max }$, maximal uptake rate; $Y$, yield. 
in which $J_{\mathrm{a}}$ is the rate of anabolism $\left(-J_{\mathrm{a}}=\mu\right), L_{\mathrm{aa}}$ and $L_{\mathrm{ac}}$ are constants, and $\Delta G_{\mathrm{a}}$ and $\Delta G_{\mathrm{c}}$ the free energies of the overall reactions of anabolism and catabolism, respectively. Ideally speaking, under conditions of catabolic limitation the substrates for anabolism are present in excess and the term $L_{\mathrm{aa}} \cdot \Delta G_{\mathrm{a}}$ will be independent of substrate concentration [see Westerhoff (1983) for a thorough analysis]. The rate of growth $\left(-J_{\mathrm{a}}\right)$ will be determined by variations in the term $L_{\mathrm{ac}} \cdot \Delta G_{\mathrm{c}}$. This term has the form:

$$
L_{\mathrm{ac}} \cdot \Delta G_{\mathrm{c}}=L_{\mathrm{ac}} \cdot\left(\Delta G_{\mathrm{c}}^{0}+R T \cdot \ln \frac{\text { [products] }}{\bar{s}}\right)
$$

Thus, equations (2) and (3) predict that the growth rate shows a linear relationship with the logarithm of $\bar{s}$.

In order to investigate the applicability of the two descriptions, we set out to determine $\bar{s}$ in glucose-limited chemostat cultures of 'Klebsiella aerogenes' (K. pneumoniae) at different growth rates. However, it was then observed that the criterion generally used to assess the achievement of a steady state - i.e. constancy of dry weight - was insufficient. Although constant cell density was established within the expected time (six to seven volume throughputs after the dilution rate was set at $0 \cdot 2 \mathrm{~h}^{-1}$ ), the glucose concentration continued to decrease significantly over a prolonged period. This suggested a physiological change of the cells, possibly due to adaptation to a glucose-limited environment. In this paper we report the effect of prolonged glucose-limited growth conditions on both the anabolic and the catabolic potential of $K$. pneumoniae.

\section{METHODS}

Organism. 'Klebsiella aerogenes' ( $K$. pneumoniae) NCTC 418 was used throughout all experiments. Cells were maintained on nutrient agar (Gibco) and subcultured every two weeks.

Growth conditions. Cells were grown in glucose-limited chemostat cultures $(500 \mathrm{ml})$, in most experiments at a dilution rate of $0.22 \pm 0.02 \mathrm{~h}^{-1}$, a temperature of $35 \pm 1{ }^{\circ} \mathrm{C}$, and $\mathrm{pH} 6.9 \pm 0 \cdot 1$. The cultures were sparged with air $\left(30 \pm 31 \mathrm{~h}^{-1}\right)$ and stirred at 1000 r.p.m. Simple salts media were used as specified by Evans $e$ al. (1970), with the exception that no trace elements and no citrate were added. Glucose $\left(2 \mathrm{gl}^{-1}\right)$ was added as the carbon and energy source. Batch cultures contained a medium similar to that used for chemostat cultures with $50 \mathrm{~mm}$-phosphate added as buffer $\left(\mathrm{pH} 6 \cdot 9 \pm 0 \cdot 1,35 \pm 1{ }^{\circ} \mathrm{C}\right)$. The cultures were aerated with filtered air.

Sampling. Samples $(1-2 \mathrm{ml})$ were taken by removing culture fluid directly from the chemostat through a Millipore filter. Cells were separated from the supernatant immediately after withdrawal from the chemostat culture.

Determinations. Dry weight of chemostat cultures (always $0.95 \pm 0.03 \mathrm{~g} \mathrm{l}^{-1}$ ) was determined by the method of Herbert et al. (1965). Glucose in the culture fluid of glucose-limited chemostat cultures was assayed fluorimetrically with the hexokinase/glucose-6-phosphate dehydrogenase system (Williamson \& Corkey, 1969). Glucose in the culture fluid of batch cultures used to determine the maximal uptake rate was assayed with glucose oxidase, using Glox reagent (AB Kabi, Stockholm, Sweden). $\mu_{\max }$ was determined in two different ways. In the experiments described in Fig. 3, a culture $(50 \mathrm{ml})$ was inoculated with cells from the chemostat and the change in optical density at $540 \mathrm{~nm}$ was followed in time. The initial $\mathrm{OD}_{540}$ was $0.05 \pm 0.02$ as measured against medium. The culture was diluted when the $\mathrm{OD}_{540}$ reached 0.6-0.7 until a constant growth rate was observed. In the experiment described in Fig. 4, a glucose-limited chemostat culture of $K$. pneumoniae was maintained for a long period at $D=0.21 \mathrm{~h}^{-1}$, then the glucose concentration of the influent medium was rapidly increased (to $5 \mathrm{~g} \mathrm{l}^{-1}$ ) and the dilution rate at the same time was set at $D=1.38 \mathrm{~h}^{-1}$. Samples of the effluent were taken to measure $\mathrm{OD}_{540}$, protein concentration, cell number and relative cell size (the latter was determined in the multichannel analyser of a Coulter counter).

The maximal uptake rate of glucose was determined by measuring the disappearance of glucose (initial concentration $10 \mathrm{~mm}$ ) from a $1: 1$ mixture of phosphate buffer $(50 \mathrm{mM}, \mathrm{pH} 6.9)$ and chemostat culture fluid at $35^{\circ} \mathrm{C}$.

\section{RESULTS AND DISCUSSION}

Growth parameters such as yield $(Y)$ and product formation usually become constant rather soon after nutrient-limited conditions are established with chemostat cultures. Traditionally, it is assumed that six to seven volume throughputs are sufficient to achieve a new steady state after some perturbation has been introduced. This also seemed to hold for aerobic glucose-limited cultures of $K$. pneumoniae. Setting the dilution rate to $0 \cdot 2 \mathrm{~h}^{-1}$ after growth in the chemostat had started resulted in a constant dry weight within $40 \mathrm{~h}$. Since the residual glucose concentration 


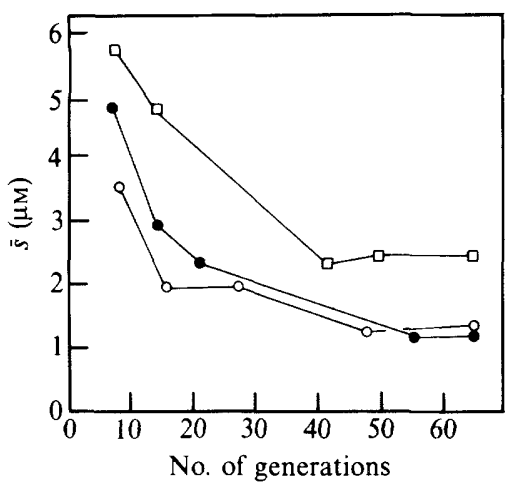

Fig. 1

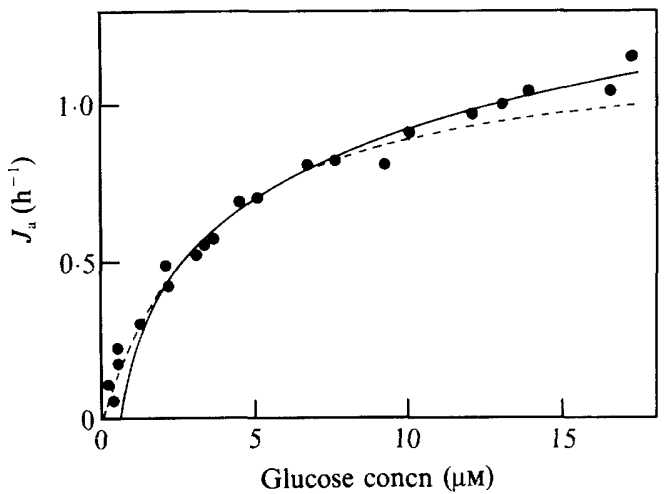

Fig. 2

Fig. 1. Effect of prolonged growth of $K$. pneumoniae on $\bar{s}$ in glucose-limited chemostat cultures $\left(D=0.2 \pm 0.02 \mathrm{~h}^{-1}\right)$. Different symbols indicate independent chemostat runs. Generations $=0$ indicates the moment where $Y_{\mathrm{Gle}}$ has become constant.

Fig. 2. Relationship between the growth rate of $K$. pneumoniae in glucose-limited chemostat culture and the steady-state concentration of glucose $(\bar{s})$. The best-fit logarithmic $(-)$ or hyperbolic curves $(-\cdot-)$ through the experimental points are indicated.

had then decreased to a value of much less than $0.1 \mathrm{~mm}$ (i.e. had become negligible in comparison with the glucose-input concentration, $11 \mathrm{mM}), Y_{\mathrm{Gic}}$ could be considered to be constant. However, no true steady state was achieved since precise glucose determinations showed that $\bar{s}$ continued to decrease for a considerable period. Thus, with a culture growing at a dilution rate of $0 \cdot 2 \mathrm{~h}^{-1}$, it took 40 to 60 generations to establish a constant value for $\bar{s}$ after a constant value for $Y_{\mathrm{Glc}}$ had been attained (Fig. 1). Similar results were obtained with cultures growing at $D=0.5 \mathrm{~h}^{-1}$ (data not shown).

To answer the primary question, whether the dependence of the growth rate on $\bar{s}$ is hyperbolic or logarithmic, we allowed a period of at least 50 generations to establish the steady state. Results of such an experiment are shown in Fig. 2. It is clear that both the best-fitting logarithmic and the best-fitting hyperbolic curves describe the data within experimental error (cf. Schulze \& Lipe, 1964). Apparently, this type of experiment cannot discriminate between the two descriptions. It is worth noting that the apparent affinity for glucose derived from the bestfit hyperbola $\left(K_{\mathrm{s}}=2 \mu \mathrm{M}\right)$, corresponds much more closely to the apparent affinity for known glucose-uptake systems of Enterobacteriaceae, such as the PEP:sugar phosphotransferase system, than that reported earlier by Schulze \& Lipe (1964) $\left(K_{\mathrm{s}}=200 \mu \mathrm{M}\right)$.

According to the Monod equation (equation 1), the residual glucose concentration at a given rate is determined by the value of $K_{\mathrm{s}}$ and $\mu_{\max }$. Therefore, a decrease in $\bar{s}$ may either be due to an increase in $\mu_{\max }$ or to a decrease in $K_{\mathrm{s}}$ (or to both). We have tested the former possibility by measuring $\mu_{\max }$ (in batch cultures) of organisms that had been grown for various periods in glucose-limited chemostat cultures at constant dilution rates. In all cases, a significant increase in $\mu_{\max }$ was observed (Fig. 3). Again, it took about 40 to 60 generations to establish a constant $\mu_{\max }$.

Determination of growth rates by measuring optical density can be misleading, because changes in cell morphology may render a proportional relationship between optical density and cell number invalid. However, there are two arguments to support the applicability of optical density measurement in our case. In the first place, we have been able to maintain a chemostat culture running at a dilution rate of $1.22 \mathrm{~h}^{-1}$ for $48 \mathrm{~h}$ with cells having a $\mu_{\max }$ of $1.25 \mathrm{~h}^{-1}$ in batch culture. Populations with a $\mu_{\max }$ value of $1.00 \mathrm{~h}^{-1}$ could not be maintained in chemostats set at a dilution rate of $1.20 \mathrm{~h}^{-1}$. This suggests that the determination of $\mu_{\max }$ would yield a realistic value. Secondly, we verified that the increase in optical density was proportional to the increase in cell number during glucose-sufficient growth (Fig. 4). Apart from some anomalies during the 


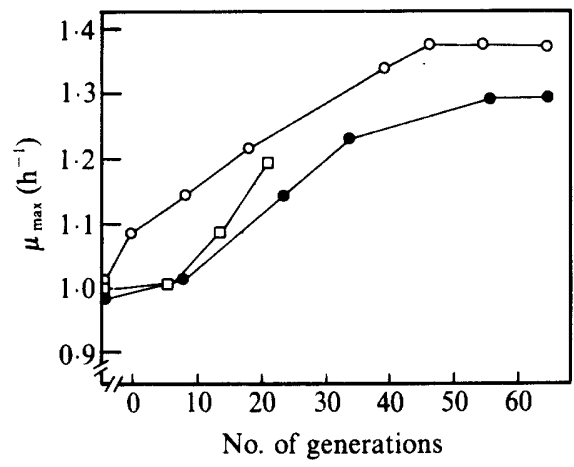

Fig. 3

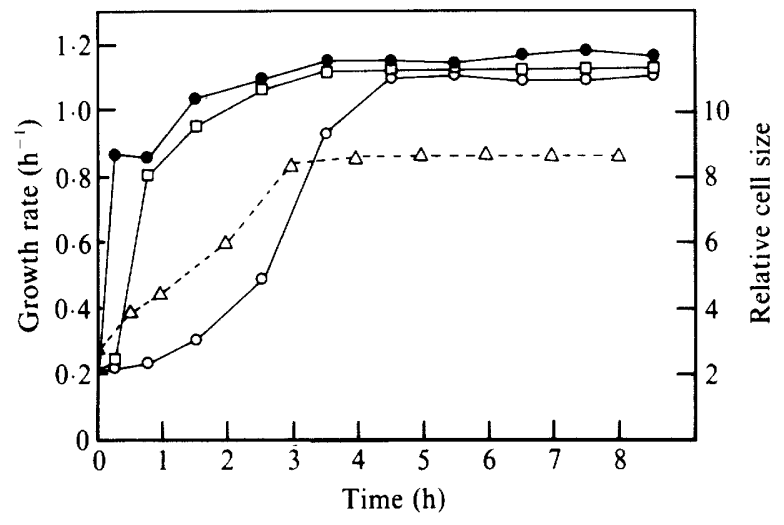

Fig. 4

Fig. 3. Effect of prolonged growth of $K$. pneumoniae in glucose-limited chemostat culture $(D=0 \cdot 22 \pm$ $\left.0.02 \mathrm{~h}^{-1}\right)$ on $\mu_{\max }$. Different symbols indicate independent chemostat runs. Generations $=0$ indicates the moment where $Y_{\mathrm{Glc}}$ has become constant. $\mu_{\max }$ before generations $=0$ was determined with cells pregrown on nutrient agar slopes.

Fig. 4. Comparison of the rate of growth of $K$. pneumoniae under glucose-sufficient conditions, as derived from number of cells $(O)$, protein $(\square)$, or $O_{540}(\odot)$. The relative size of the cells $(\triangle)$ was determined with a Coulter counter:

initial phase of growth, which may have been due to changes in cell volume, the rates of increase in cell number, optical density and protein concentration were equal within experimental error.

An increase in $\mu_{\max }$ implies an increase in maximal flux through the various metabolic pathways, including the anabolic and catabolic carbon flow. To verify this, cells were taken from glucose-limited chemostat cultures and suspended in a phosphate buffer containing a saturating concentration of glucose. The disappearance of glucose from the medium was then followed with time. Since no accelerated growth occurred during the first $20 \mathrm{~min}$, these experiments allowed the calculation of the maximal specific rate of uptake of glucose (Fig. 5). There was a significant increase in the glucose uptake capacity, suggesting an increased catabolic capacity, when cells were cultured under glucose-limited conditions for a prolonged period.

It is clear from the results presented here that under continuously glucose-limited conditions a shift occurred in the population towards organisms that possess an enhanced metabolic potential for the carbon and energy source. The question then arises as to the mechanism underlying this phenomenon. Phenotypic adaptation to nutrient-limited conditions is well documented (for a review see Harder \& Dijkhuizen, 1983), but it has to be realized that the constancy of the environment in chemostat cultures may also result in the appearance of mutants that are better suited to grow in that particular environment. An important observation in this connection was that populations with an increased $\mu_{\max }$ did not lose this potential when transferred to plates containing minimal medium plus $5.5 \mathrm{~mm}$-glucose: single colonies were grown in batch cultures and always expressed a maximal growth rate of $1.20 \mathrm{~h}^{-1}$. However, when these cells were cultured on complex medium and subsequently transferred to minimal medium ( $+5.5 \mathrm{mM}$ glucose), the $\mu_{\max }$ had decreased to $1.00 \mathrm{~h}^{-1}$ again, suggesting that the elevated metabolic potential is maintained only on minimal glucose medium. It would be interesting to test whether the cells with the decreased $\mu_{\max }$ have the same long-term adaptation pattern. We have also observed an apparent variability in our laboratory culture. Sometimes the establishment of $\bar{s}$ was relatively rapid, i.e. it occurred in less than 10 generations. In all cases where we have measured the time course of $\bar{s}$ and $\mu_{\max }$, their steady state was reached with approximately the same kinetics. 


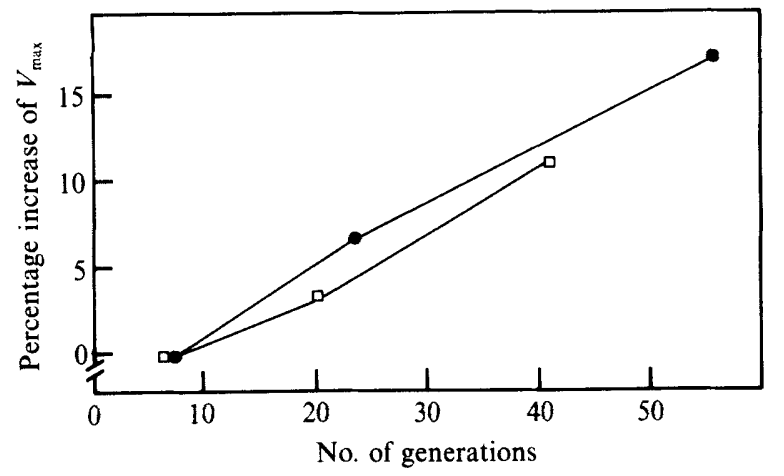

Fig. 5. Effect of prolonged growth of $K$. pneumoniae in glucose-limited chemostat culture $(D=0.22 \pm$ $\left.0.02 \mathrm{~h}^{-1}\right)$ on $V_{\max }$. Different symbols indicate independent chemostat runs. Generations $=0$ indicates the moment where $Y_{\mathrm{Glc}}$ has become constant. Values are relative to the first value.

The importance of chemostat cultures in fundamental research resides in the fact that this technique allows growth of micro-organisms under constant and well-defined conditions. The advantage is easily appreciated when it is realized that microbial behaviour is highly dependent on the environment. However, constancy of environment has one potential drawback in that it results in constant selective pressure on the micro-organisms. Culturing organisms under a constant nutrient-limited growth condition, as occurs in chemostat cultures, may lead to the selection of organisms that are extremely well adapted to that particular environment. Our results indicate that populations that are exposed to a nutrient-limited environment can be affected in their physiological properties. Similar results have been obtained with other microbial species grown under various nutrient-limited conditions (Collins et al., 1976; Höfle, 1983; Kyslik \& Sikyta, 1984). In general, it can be concluded that the long-term changes in physiology are accompanied by enhanced maximal metabolic rates or increased affinities for the growth-limiting substrate. These shifts can be rationalized in terms of the existing ideas about competition in chemostat cultures (Powell, 1958; Dykhuizen \& Hartl, 1981, 1983; Harder et al., 1977; Tempest et al., 1983). From the classical chemostat theory, it can be derived that organisms that are able to maintain a certain growth rate at a lower concentration of the substrate that limits growth than their competitors will be successful simply because the ambient substrate concentration is lowered. As a consequence, the growth rate of the competitor would become lower than the dilution rate and this population will eventually wash out from the culture. Hence, a high $\mu_{\max }$ is an advantageous property under nutrient-limited conditions, despite the fact that these high rates will in nature hardly ever be attained: the potential to grow faster allows for specific growth rates to occur at lower substrate concentrations.

A second possibility to increase the ability to scavenge the limiting nutrient from the environment is to invoke an altered or completely different uptake system with a high affinity for the substrate, i.e. a lowering of $K_{\mathrm{s}}$. Again, this will result in the ability to maintain a given growth rate at lower substrate concentration. An example of this type of adaptation may be given by the work of Collins et al. (1976), who demonstrated that in alanine-limited chemostat cultures of Escherichia coli, the proton/alanine stoichiometry during alanine transport increased from 2 to 4 in about 25 generations.

Applying equation (1) to the results presented here suggests that an increase in $\mu_{\max }$ alone cannot account for the decrease in $\bar{s}$. When equation (1) is rewritten as follows

$$
\bar{s}=K_{\mathrm{s}} \cdot \frac{\mu}{\mu_{\max }-\mu}
$$

it can be seen that $\bar{s}$ has to diminish hyperbolically with increasing $\mu_{\max }$. The decrease in $\bar{s}$ in our experiments, combined with the observed increase in $\mu_{\max }$, suggests that $K_{\mathrm{s}}$ has to become half 
its initial value in the course of 50 generations, i.e. decrease from $4 \mu \mathrm{M}$ to $2 \mu \mathrm{M}$. This is not necessarily due to a qualitative change in the uptake system, but may be caused by the fact that $K_{\mathrm{s}}$ is an apparent value that reflects the overall metabolic affinity. This apparent value may well be influenced by changes in the $V_{\max }$ value of one reaction in the total metabolic pathway.

The question arises whether the observed changes are due to phenotypic or genotypic adaptations. Phenotypic adaptation has been reported frequently and generally includes derepression/induction of high-affinity uptake systems for the limiting nutrient. In contrast to the data presented here, this always occurs rapidly after the cultures have become nutrient limited. With the long-term changes reported by us, this type of adaptation seems less likely because it would imply that the extent of induction is dependent on small differences in glucose concentration.

As has been pointed out, genotypic adaptation is to be expected as well, and many theoretical studies have been published on this subject (Powell, 1958; Harder et al., 1977; Dykhuizen, 1978; Dykhuizen \& Hartl, 1983). The occurrence of mutants in chemostat cultures has been studied extensively (e.g. Cox \& Gibson, 1974; Hartl et al., 1983; Chao \& McBroom, 1985) and the main conclusion seems to be that bacterial strains endowed with a high mutation rate (e.g. when they possess transposable elements) will eventually outcompete organisms with a lower mutation rate (Chao \& McBroom, 1985). The rationale for this is that with the former strains beneficial mutations will occur at an increased rate.

If our observations result from genotypic adaptation, a difficulty arises in calculations of mutation rates. First, it should be realized that the simultaneous presence of two strains with different $\mu_{\max }$ and/or $K_{\mathrm{s}}$ values results in an $\bar{s}$ that is dependent on time. So, the actual growth rates of the two organisms become variable. This computational complication has been solved by Powell (1958). A second problem arises because the number of faster-growing cells is not only determined by the differences in $\mu_{\max }$ and $K_{\mathrm{s}}$, but also by the mutation rate. Finally, it is as yet not clear whether the determined $\mu_{\max }$ is the average of a rapidly growing and a slowly growing population, or the average of a number of populations with intermediate values for $\mu_{\max }$. A simplified calculation [based on the equations developed by Powell (1958) and assuming that there are only two populations with different $\mu_{\max }$ present] shows that the population at the time when $Y_{\mathrm{Glc}}$ becomes constant has to contain about $1 \%$ of the faster-growing cells. Of course, this is an unrealistically high value. However, it can be argued that this percentage is grossly overestimated. First, neither a decrease in the apparent $K_{\mathrm{s}}$ nor the contribution of the mutational rate to the 'high $\mu_{\max }$ ' population has been taken into account. Second, before $Y_{\mathrm{Glc}}$ has become constant, selection may have taken place during the growth phase after inoculation. For instance, it is possible that the lag time after inoculation of the parental strain is significantly longer than that of the mutant.

Our data may suggest that in chemostat cultures mutants appear that are especially fit to grow under the ambient growth conditions. From the results it can be concluded that such mutations are unstable since $\mu_{\max }$ is diminished after growth in complex medium. A possible explanation of this phenomenon could be that the selected mutants arise by the mechanism of tandem duplication (Anderson et al., 1976) of relevant genes (e.g. coding for the glucose-uptake system). However, we have no evidence for this suggestion. Further studies on variations in DNA content and composition would be necessary to verify this hypothesis.

An important consequence of the appearance of mutants in chemostat cultures is that the attainment of a steady state may take a considerably longer time than is expected from macroscopic parameters such as $Y_{\mathrm{Glc}}$ and product-formation rates. We therefore believe that the most important conclusion is that great care has to be taken when defining a steady state. We realize that our results may be confined to the special case of glucose-limited conditions. For this reason, we are currently investigating the behaviour of ammonia-limited cultures.

Supported by grants from the Netherlands Organization for the Advancement of Pure Research (ZWO) and the Netherlands Technology Foundation (STW). 


\section{REFERENCES}

ANDerson, R. P., Miller, C. G. \& Roth, J. R. (1976). Tandem duplications of the histidine operon observed following generalized transduction in Salmonella typhimurium. Journal of Molecular Biology 105 , 201-218.

ChaO, L. \& McBroom, S. M. (1985). Evolution of transposable elements: an IS10 insertion increases fitness in Escherichia coli. Molecular Biology and Evolution 2, 359-369.

Collins, S. H., JaRvis, A. W., Lindsay, R. J. \& Hamilton, W. A. (1976). Proton movements coupled to lactate and alanine in Escherichia coli: isolation of mutants with altered stoichiometry in alanine transport. Journal of Bacteriology 126, 12321244.

Cox, E. C. \& Gibson, T. C. (1974). Selection for high mutation rates in chemostats. Genetics 77, 169-184.

Dam, K. van, Mulder, M. M., Teixeira de Mattos, M. J. \& WesterhofF, H. V. (1987). A thermodynamic view of bacterial growth. In Physiological Models (CRC Mathematical Models of Microbiology) (in the Press). Edited by J. Prosser \& M. J. Bazin. Boca Raton: CRC Press.

DYKhuizen, D. E. (1978). Selection for tryptophan auxotrophs of Escherichia coli in glucose-limited chemostats as a test of the energy conservation hypothesis of evolution. Evolution 32, 125-150.

Dykhuizen, D. E. \& HaRTL, D. L. (1981). Evolution of competitive ability in Escherichia coli. Evolution 35, 581-594.

Dykhuizen, D. E. \& HARTL, D. L. (1983). Selection in chemostats. Microbiological Reviews 47, 150-168.

Evans, C. G. T., Herbert, D. \& Tempest, D. W. (1970). The continuous culture of microorganisms. 2. Construction of a chemostat. Methods in Microbiology 2, 277-327.

Harder, W. \& Dijkhuizen, L. (1983). Physiological responses to nutrient limitation. Annual Review of Microbiology 37, 1-23.

Harder, W., Kuenen, J. G. \& Matin, A. (1977). Microbial selection in continuous culture. Journal of Applied Bacteriology 43, 1-24.
Hartl, D. L., Dykhuizen, D. E., Miller, R. D., Green, L. \& Framond, J. DE (1983). Transposable element IS50 improves growth rate of $E$. coli cells without transposition. Cell 35, 503-510.

Herbert, D., Phipps, P. J. \& Tempest, D. W. (1965). The chemostat: design and instrumentation. Laboratory Practice 14, 1150-1161.

HöFLE, M. (1983). Long term changes in chemostat cultures of Cytophaga johnsonae. Applied and Environmental Microbiology 46, 1045-1053.

KYSLIK, P. \& SikYTA, B. (1984). Population changes in a culture of Escherichia coli K12 1EA growing in a ribitol-limited chemostat. Biotechnology Letters 6, 25-30.

MONOD, J. (1950). La technique de culture continue; theorie et applications. Annales de l'Institut Pasteur 79, $390-410$.

Powell, E. O. (1958). Criteria for the growth of contaminants and mutants in continuous culture. Journal of General Microbiology 18, 259-268.

SChulze, K. L. \& LIPE, R. S. (1964). Relationship between substrate concentration, growth rate and respiration rate of Escherichia coli in continuous culture. Archiv für Mikrobiologie 48, 1-20.

Tempest, D. W., Neijssel, O. M. \& Zevenboom, W. (1983). Properties and performances of microorganisms in laboratory culture; their relevance to growth in natural ecosystems. Symposia of the Society for General Microbiology 34, 119-152.

WESTERHOFF, H. V. (1983). Mosaic non-equilibrium thermodynamics and (the control of) biological freeenergy transduction. Thesis, University of Amsterdam.

Westerhoff, H. V., LOLKEMa, J. S., OtTo, R. \& HellingwerF, K. J. (1982). Thermodynamics of growth. Non-equilibrium thermodynamics of bacterial growth. The phenomenological and the mosaic approach. Biochimica et biophysica acta 683, 181-220.

Williamson, J. R. \& CORKEY, B. E. (1969). Assays of intermediates of the citric acid cycle and related compounds by fluorometric enzyme methods. Methods in Enzymology 13, 434-513. 